\title{
Numerical Study of the Influence of Heat Source on Stagnation Point Flow towards a Stretching Surface of a Jeffrey Nanoliquid
}

\author{
G. K. Ramesh ${ }^{1,2}$ \\ ${ }^{1}$ Department of Studies and Research in Mathematics, Kuvempu University, Shankaraghatta, Shimoga, Karnataka 577 451, India \\ ${ }^{2}$ Department of Mathematics, SEA College of Engineering and Technology, K. R. Puram, Bengaluru, Karnataka 560 049, India
}

Correspondence should be addressed to G. K. Ramesh; gkrmaths@gmail.com

Received 5 August 2014; Accepted 27 February 2015

Academic Editor: Oronzio Manca

Copyright (c) 2015 G. K. Ramesh. This is an open access article distributed under the Creative Commons Attribution License, which permits unrestricted use, distribution, and reproduction in any medium, provided the original work is properly cited.

\begin{abstract}
An analysis is carried out to study the flow of Jeffrey fluid near a stagnation point towards a permeable stretching sheet. In particular, we investigate the effect of temperature dependent internal heat generation or absorption in the presence of nanoparticles. The governing system of partial differential equations is transformed into ordinary differential equations, which are then solved numerically using the fourth-fifth-order Runge-Kutta-Fehlberg method. Comparisons with previously published work on special cases of the problem are performed and found to be in excellent agreement. The results of the governing parametric study are shown graphically and the physical aspects of the problem are highlighted and discussed.
\end{abstract}

\section{Introduction}

Investigations on boundary layer flow and heat transfer of non-Newtonian fluids are increasing substantially due to the large number of practical applications in industrial and manufacturing processes. Examples of such applications are drilling muds, plastic polymers, optical fibers, hot rolling paper production, metal spinning, cooling of metallic plates in cooling baths, and many others. In the past, investigators proposed different non-Newtonian models because a single model cannot predict all the features of non-Newtonian materials. There is one subclass of non-Newtonian fluids known as Jeffrey fluid which has attracted much attention from the researchers in view of its simplicity. This fluid model is capable of describing the characteristics of relaxation and retardation times $[1,2]$. The investigation of flow due to a stretching sheet has been intentional because of this flow's various industrial applications, such as in the manufacturing of polymer sheets, filaments, and wires. During the manufacturing process, the moving sheet is assumed to stretch on its own plane, and the stretched surface interacts with the ambient fluid both mechanically and thermally. Stretching and shrinking can occur in a variety of materials each having a different strength, stretching transparency, and luster. Initially, Sakiadis [3] introduced the concept of a boundary layer flow over a stretching surface. Crane [4] modified the idea introduced by Sakiadis and extended this idea for both linear and exponentially stretching sheets.

Flow in the neighborhood of a stagnation point in a plane was first studied by Hiemenz [5] and Mahapatra et al. [68 ] investigated the magnetohydrodynamic stagnation point flow towards a stretching sheet; they have shown that the velocity at a point decreases/increases with increase in the magnetic field when the free stream velocity is less/greater than the stretching velocity. Also they have studied the temperature distribution when the surface at constant temperature and constant heat fluxes. Further they have extended their work on power-law fluid and discussed the unique solutions of stagnation point flow of a power-law fluid towards a stretching surface. The study of heat source/sink effects on heat transfer is very important because its effects are crucial in controlling the heat transfer. Postelnicu et al. [9] examined the effect of variable viscosity on forced convection flow past a horizontal flat plate in a porous medium with internal heat generation, but in heat generation part they considered only space dependent heat source. Abel et al. [10] analyzed 
the non-Newtonian viscoelastic boundary layer flow of Walter's liquid B past a stretching sheet, taking account of nonuniform heat source.

Aforementioned studies were primarily concerned with the laminar flow of a clear fluid. In the recent past a new class of fluids, namely, nanofluids, has attracted the attention of the science and engineering community because of the many possible industrial applications of these fluids. Nanotechnology is an emerging science that is finding extensive use in industry due to the unique chemical and physical properties that the nanosized materials possess. These fluids are colloidal suspensions, typically metals, oxides, carbides, or carbon nanotubes in a base fluid. The term nanofluid was coined by Choi [11] in his seminal paper presented in 1995 at the ASME Winter Annual Meeting. It refers to fluids containing a dispersion of submicronic solid particles (nanoparticles) with typical length on the order of 1-50 nm. Kuznetsov and Nield [12] analytically studied the natural convective boundary layer flow of a nanofluid past a vertical plate. In a recent paper Khan and Pop [13] studied for the first time the problem of laminar fluid flow resulting from the stretching of a flat surface in a nanofluid. Mustafa et al. [14] investigated the stagnation point flow of a nanofluid towards a stretching surface using homotopy analysis method. Alsaedi et al. [15] examined the influence of heat generation/absorption on the stagnation point flow of nanofluid towards a linear stretching surface. Rahman et al. [16] investigated the dynamics of the natural convection boundary layer flow of water based nanofluids over a wedge in the presence of a transverse magnetic field with internal heat generation or absorption with the help of Matlab software. Nandy and Mahapatra [17] analyzed the effects of velocity slip and heat generation/absorption on magnetohydrodynamic stagnation point flow and heat transfer over a stretching/shrinking surface and obtained the solution numerically using fourth-order Runge-Kutta method with the help of shooting technique. Different from a stretching sheet, it was found that the solutions for a shrinking sheet are nonunique. Makinde et al. [18] studied the combined effects of buoyancy force, convective heating, Brownian motion, and thermophoresis on the stagnation point flow and heat transfer of an electrically conducting nanofluid towards a stretching sheet under the influence of magnetic field. Effect of magnetic field on stagnation point flow and heat transfer due to nanofluid towards a stretching sheet have been investigated by Ibrahim et al. [19]. Recently Ramesh and Gireesha [20] investigated the boundary layer flow of Maxwell fluid over a stretching surface with convective boundary condition in the presence of nanoparticles and heat source/sink effect. Nadeem et al. $[21,22]$ reported the numerical solutions of non-Newtonian nanofluid flow over a stretching sheet using the Jeffrey fluid model. Further they obtained the analytic solution for nonorthogonal stagnation point flow of a non-Newtonian nanofluid towards a stretching surface with heat transfer; here they use the second-grade model. Nadeem et al. [22] studied the natural convection boundary layer flow over a downward-pointing vertical cone in a porous medium saturated with a non-Newtonian nanofluid in the presence

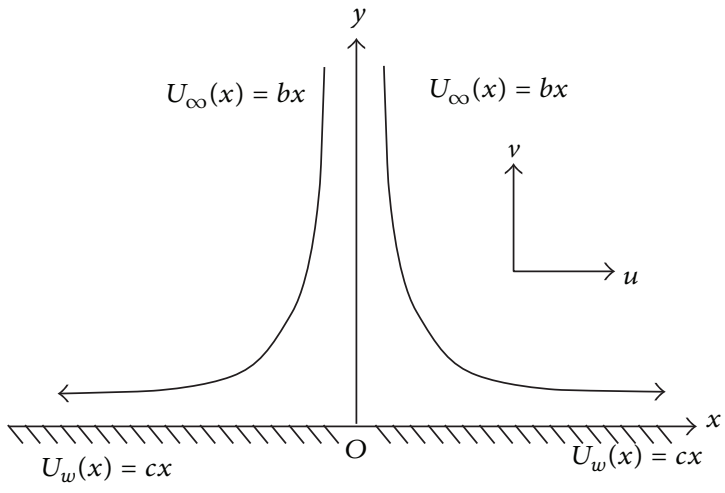

FIGURE 1: Schematic diagram of the flow.

of heat generation or absorption and they used power-law model. Some interesting recent investigations related to the topic are presented in [23-25].

In this paper, we study the behaviour of the stagnation point flow towards a stretching sheet with the effects of heat source/sink and suction in the presence of nanoparticles. Similarity transforms are presented for this problem, and nondimensionalized equations are addressed numerically. Graphical results for various values of the parameters are presented to gain thorough insight towards the physics of the problem. To the best of my knowledge, this problem has not been studied before.

\section{Mathematical Analysis}

Consider the flow of an incompressible Jeffrey fluid in the region $y>0$ driven by a stretching surface located at $y=0$ with a fixed stagnation point at $x=0$. The stretching velocity $U_{w}(x)$ and the ambient fluid velocity $U_{\infty}(x)$ are assumed to vary linearly from the stagnation point; that is, $U_{w}(x)=c x$ and $U_{\infty}(x)=b x$, where $c$ and $b$ are constant as shown in Figure 1. The problem under consideration is governed by the following boundary layer equations of Jeffrey fluid and nanoparticles and heat generation or absorption are given by Nadeem et al. [21]:

$$
\frac{\partial u}{\partial x}+\frac{\partial v}{\partial y}=0
$$

$$
\begin{aligned}
& \frac{1+\lambda}{v}\left(u \frac{\partial u}{\partial x}+v \frac{\partial u}{\partial y}\right) \\
& =\frac{\partial^{2} u}{\partial y^{2}}+U_{\infty} \frac{d U_{\infty}}{d x} \\
& \quad+\lambda_{1}\left(u \frac{\partial^{3} u}{\partial x \partial y^{2}}+v \frac{\partial^{3} u}{\partial y^{3}}-\frac{\partial u}{\partial x} \frac{\partial^{2} u}{\partial y^{2}}+\frac{\partial u}{\partial y} \frac{\partial^{2} u}{\partial x \partial y^{2}}\right),
\end{aligned}
$$




$$
\begin{aligned}
u \frac{\partial T}{\partial x}+ & v \frac{\partial T}{\partial y} \\
=\alpha & \left(\frac{\partial^{2} T}{\partial x^{2}}+\frac{\partial^{2} T}{\partial y^{2}}\right) \\
+ & \tau\left\{D_{B}\left(\frac{\partial C}{\partial x} \frac{\partial T}{\partial x}+\frac{\partial C}{\partial y} \frac{\partial T}{\partial y}\right)\right. \\
& \left.+\left(\frac{D_{T}}{T_{\infty}}\right)\left[\left(\frac{\partial T}{\partial x}\right)^{2}+\left(\frac{\partial T}{\partial y}\right)^{2}\right]\right\} \\
+ & \frac{Q_{0}}{\rho_{f} c_{p}}\left(T-T_{\infty}\right), \\
u \frac{\partial C}{\partial x}+ & v \frac{\partial C}{\partial y} \\
= & D_{B}\left(\frac{\partial^{2} C}{\partial x^{2}}+\frac{\partial^{2} C}{\partial y^{2}}\right) \\
& +\left(\frac{D_{T}}{T_{\infty}}\right)\left[\left(\frac{\partial^{2} T}{\partial x^{2}}\right)+\left(\frac{\partial^{2} T}{\partial y^{2}}\right)\right]
\end{aligned}
$$

where $u$ and $v$ are the velocity components along the $x$ - and $y$-axes, respectively. Further, $\alpha, \rho_{f}, \rho_{p}, v, T$, and $T_{\infty}$ are, respectively, the thermal diffusivity, density of the base fluid, density of the particles, kinematic viscosity of the fluid, fluid temperature, and ambient fluid temperature. $\lambda$ and $\lambda_{1}$ are ratios of relaxation to retardation times and retardation time, $Q_{0}$ is the dimensional heat generation/absorption coefficient, $D_{B}$ is the Brownian diffusion coefficient, $D_{T}$ is the thermophoresis diffusion coefficient, and $c_{p}$ is the specific heat at constant pressure. Here $\tau$ is the ratio of the effective heat capacity of the nanoparticle material to the heat capacity of the ordinary fluid and $C$ is the nanoparticle volume fraction.

The associated boundary conditions for the present problem are

$$
\begin{array}{r}
u=U_{w}(x), \quad v=-V_{w}(x), \quad T=T_{w}, \quad C=C_{w} \\
\text { at } y=0, \\
u \longrightarrow U_{\infty}(x), \quad v \longrightarrow 0, \quad \frac{\partial u}{\partial y} \longrightarrow 0, \quad T \longrightarrow T_{\infty}, \\
C \longrightarrow C_{\infty} \quad \text { as } y \longrightarrow \infty,
\end{array}
$$

where $U_{w}(x)=c x$ is the stretching sheet velocity, $c>0$; this is known as stretching rate. $T_{w}$ and $C_{w}$ are the temperature of fluid and nanoparticles fraction at wall, and $C_{\infty}$ is the ambient nanoparticle volume fraction.

The specific forms of the stretching velocity and the surface temperature and concentration are chosen to allow the coupled nonlinear partial differential equations (1)-(4) to be converted to a set of coupled, nonlinear ordinary differential equations by the similarity transformation

$$
\begin{gathered}
\eta=\left(\frac{U_{w}}{\nu x}\right)^{1 / 2} y, \quad f(\eta)=\frac{\psi}{\left(x \nu U_{w}\right)^{1 / 2}}, \\
\theta(\eta)=\frac{T-T_{\infty}}{T_{w}-T_{\infty}}, \quad \varphi(\eta)=\frac{C-C_{\infty}}{C_{w}-C_{\infty}},
\end{gathered}
$$

where $\eta$ is the similarity variable; $f, \theta$, and $\phi$ are the dimensionless stream function, temperature, and concentration, respectively. The velocity components $u$ and $v$ in (6) automatically satisfy the continuity equation (1). In terms of $f(\eta), \theta(\eta)$, and $\phi(\eta)$ the momentum equation (2), energy equation (3), and concentration equation (4) can be written as

$$
\begin{gathered}
f_{\eta \eta \eta}+\beta\left(f_{\eta \eta}^{2}-f f_{\eta \eta \eta \eta}\right) \\
+(1+\lambda)\left(f f_{\eta \eta}-f_{\eta}^{2}\right)+A^{2}=0, \\
\theta_{\eta \eta}+\operatorname{Pr}\left(f \theta_{\eta}+N b \theta_{\eta} \phi_{\eta}+N t \theta_{\eta}^{2}+S \theta\right)=0, \\
\phi_{\eta \eta}+\operatorname{Le} f \phi_{\eta}+\frac{N t}{N b} \theta_{\eta \eta}=0 .
\end{gathered}
$$

Here $f_{\eta}(\eta)$ represents an ordinary derivative with respect to $\eta$ and the corresponding boundary conditions in the nondimensional form are

$$
\begin{array}{r}
f=f_{0}, \quad f_{\eta}=1, \quad \theta=1, \quad \phi=1 \\
\text { at } \eta=0, \\
f_{\eta} \longrightarrow A, \quad f_{\eta \eta} \longrightarrow 0, \quad \theta \longrightarrow 0, \quad \phi \longrightarrow 0 \\
\text { as } \eta \longrightarrow \infty .
\end{array}
$$

The dimensionless parameters in (7), (8), and (9) are $A=$ $c / b$ is the velocity ratio, $\beta=c \lambda_{1}$ is the Deborah number, $f_{0}=V_{w} /(\nu c)^{1 / 2}$ is suction parameter, $N b=\tau D_{B}\left(\varphi_{w}-\varphi_{\infty}\right) / \nu$ is the Brownian motion, $N t=\tau D_{T}\left(T_{w}-T_{\infty}\right) / \nu T_{\infty}$ is the thermophoresis parameter, and Le $=\nu / D_{B}$ is the Lewis number. $\operatorname{Pr}=\nu / \alpha$ is the Prandtl number; $S=Q_{0} / c \rho_{f} c_{p}$ is the heat generation $(A>0)$ or absorption $(A<0)$ parameter.

\section{Solutions for Some Special Cases}

In the limiting case of $A=0, \beta=0, \lambda=0$, and $S=$ 0 (i.e., in the absence of velocity ratio, Deborah number, ratio of relaxation to retardation times, and heat source/sink parameter ) our system of (7) and (9) reduces to those of Khan and Pop [13] (Newtonian nanofluid).

In the presence of velocity ratio parameter, when there is no Deborah number, ratio of relaxation to retardation times, and heat source/sink parameter, system of (7) and (9) reduces to those of Mustafa et al. [14] $(A \neq 0, \beta=0, \lambda=0$, and $S=$ 0 ). Further in the absence of melting effect, heat source/sink, and nanoparticles volume fraction, the equations are similar to the ones studied by Hayat [27]. 


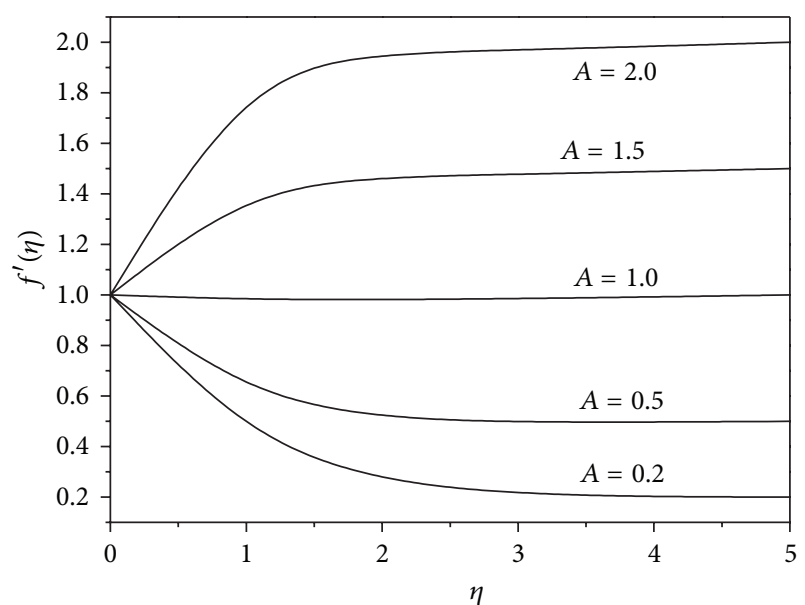

Figure 2: Velocity profile for different values of $A$.

In the absence of nanoparticles, the stagnation point flow of Jeffrey fluid and heat transfer problem degenerates. In this case, the approximate numerical solutions for the velocity field, temperature, and concentration fields are obtained.

\section{Result and Discussion}

The nonlinear coupled ordinary differential equations (7)(9) that are subject to the boundary conditions (10) have been solved numerically using a fourth-fifth-order RungeKutta-Fehlberg method to obtain the missing values of $f^{\prime \prime}(0),-\theta^{\prime}(0)$, and $-\phi^{\prime}(0)$ for some values of the governing parameters, namely, the velocity ratio parameter $A$, heat source/sink parameter $S$, Deborah number $\beta$, ratio of relaxation to retardation times parameter $\lambda$, Brownian parameter $N b$, thermophoresis parameter $N t$, Lewis number Le, and Prandtl number Pr. In order to validate the numerical results obtained, we compare our results with those reported by Mahapatra and Gupta [7], Hayat et al. [28], and Ibrahim et al. [19] for various values of $A$ as shown in Tables 1 and 2, and they are found to be in a favorable agreement.

The velocity profiles for different values of $A$ are presented in Figure 2. It is found that when the stretching velocity is less than the free stream velocity $(A>1)$, the flow has a boundary layer structure, physically saying that the straining motion near the stagnation region increases so the acceleration of the external stream increases which leads to decrease in the thickness of the boundary layer with increase in $A$. When the stretching velocity $c x$ of the surface exceeds the free stream velocity $b x(A<1)$ inverted boundary layer structure is formed and $A=1$; there is no boundary layer formation because the stretching velocity is equal to the free stream velocity. The temperature and concentration profiles for different values of $A$ with other parameters being fixed are presented in Figures 3 and 4, respectively. It can be seen from these figures that both $\theta(\eta)$ and $\phi(\eta)$ decrease with increase in $A$.

Figure 5 is plotted for velocity distribution for different values of $\beta$. When $A<1$ velocity increases with the increase

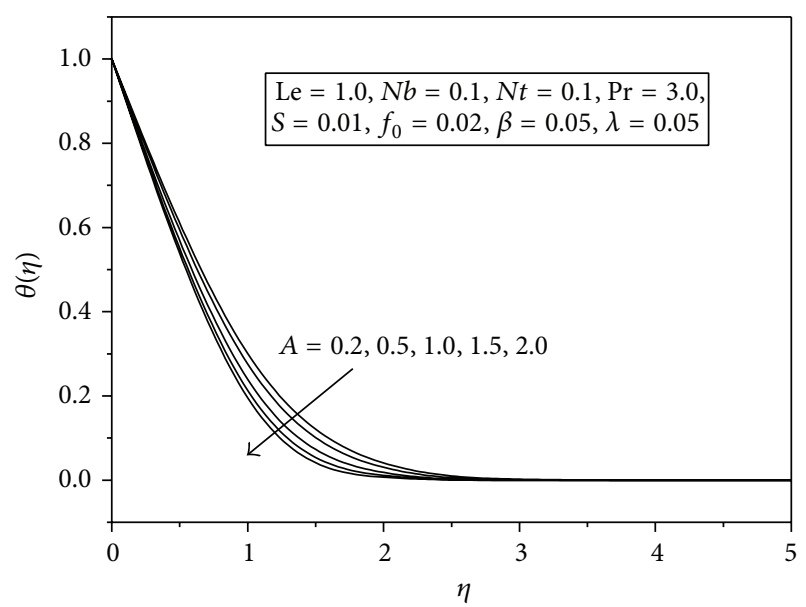

Figure 3: Temperature profile for different values of $A$.

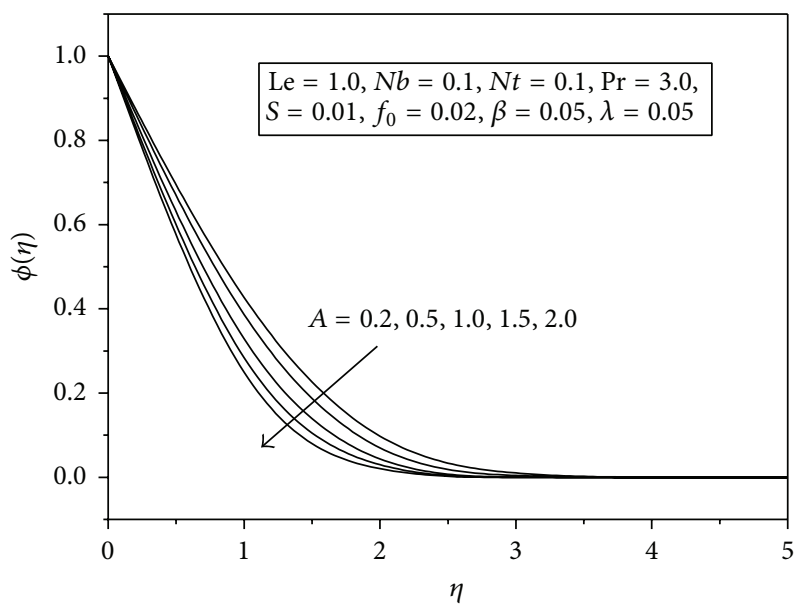

FIGURE 4: Concentration profile for different values of $A$.

of $\beta$, whereas when $A>1$ the velocity decreases with the increase of $\beta$. Similar effects can be seen in Figure 6 for various values of $\lambda$ when $A>1$, while the opposite effect can be seen when $A<1$. This is because $\beta$ is dependent upon $\lambda$ (retardation time); physically larger retardation time of any material makes it less viscous resulting in an increase in its motion. It is worth mentioning that the liquid-like behavior is associated with small Deborah number. However, large Deborah number signifies the solid-like behavior.

The graph of velocity profile $f^{\prime}$ versus $\eta$ for different values of $f_{0}$ is plotted in Figure 7. It is found that for a fixed value of $A<1$ the velocity decreases with the increase of $f_{0}$. The velocity profiles tend asymptotically to the horizontal axis; the nondimensional velocities absorb maximum at the wall. It is a fact that suction stabilizes the boundary layer growth. At $A>1$ velocity increases with the increase of $f_{0}$. Figure 8 presents temperature profiles for different values of $S$. For $S>0$ (heat source), it can be observed that the thermal boundary layer generates the energy, and this causes the temperature in the thermal boundary layer to increase 
TABLE 1: Comparison of the values of skin friction coefficient $f^{\prime \prime}(0)$ for various values of velocity ratio $A$.

\begin{tabular}{lcccc}
\hline$A$ & Mahapatra and Gupta [7] & Hayat et al. [26] & Ibrahim et al. [19] & Present study \\
\hline 0.01 & - & -0.9982 & -0.9980 & -0.99910 \\
0.1 & -0.9694 & -0.9695 & -0.9694 & -0.96941 \\
0.2 & -0.9181 & -0.9181 & -0.9181 & -0.91812 \\
0.5 & -0.6673 & -0.6673 & -0.6673 & -0.66729 \\
2.0 & 2.0175 & 2.0176 & 2.0175 & 2.01750 \\
3.0 & 4.7293 & 4.7296 & 4.7292 & 4.72923 \\
\hline
\end{tabular}

TABLE 2: Comparison of the values of Nusselt number $-\theta^{\prime}(0)$ for various values of velocity ratio $A$.

\begin{tabular}{cccccc}
\hline $\operatorname{Pr}$ & $A$ & Mahapatra and Gupta [7] & Hayat et al. [26] & Ibrahim et al. [19] & Present study \\
\hline \multirow{3}{*}{1} & 0.1 & 0.603 & 0.6021 & 0.6022 & 0.60223 \\
& 0.2 & 0.625 & 0.6244 & 0.6245 & 0.62452 \\
& 0.5 & 0.692 & 0.6924 & 0.6924 & 0.69249 \\
\hline \multirow{3}{*}{1.5} & 0.1 & 0.777 & 0.7768 & 0.7768 & 0.77687 \\
& 0.2 & 0.797 & 0.7971 & 0.7971 & 0.79712 \\
& 0.5 & 0.863 & 0.8647 & 0.8648 & 0.86475 \\
\hline
\end{tabular}

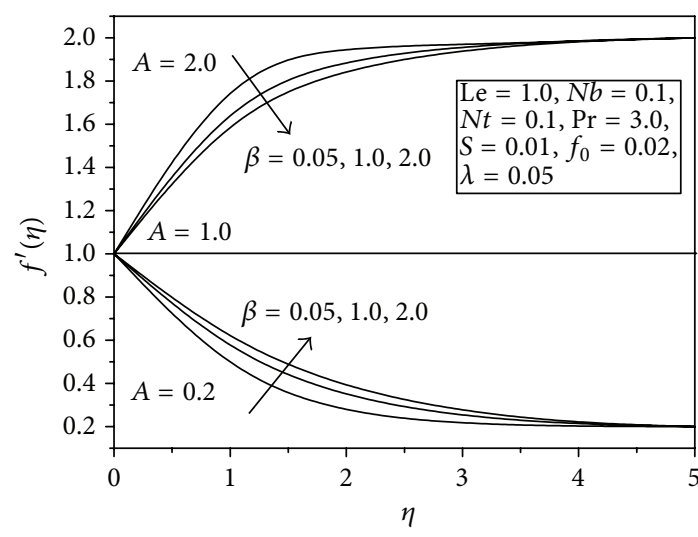

FIGURE 5: Velocity profile for different values of $\beta$.

with increase in $S$, whereas $S<0$ (heat sink) leads to a decrease in the thermal boundary layer.

Figures 9 and 10 illustrate the variation of $\theta, \phi$ with $\eta$ for various values of $N b$. It is found that the increase in the value of $N b$ is to increase the $\theta(\eta)$ in the boundary layer, whereas in concentration boundary layer reduces as $N b$ increases which thereby enhances the nanoparticles concentration at the sheet. The graphs of $N t$ on the $\theta$ and $\phi$ profile are shown in Figures 11 and 12. From these plots, it is observed that the effect of increasing values of $N t$ is to increase the temperature and concentration profiles. Figure 13 displays the effect of Le on concentration profiles. It is noted that the concentration of the fluid decreases with increase of Le, physically due to the fact that mass transfer rate increases as Le increases. It also reveals that the concentration gradient at surface of the plate increases.

Temperature and concentration profiles for the selected values of Pr are plotted in Figures 14 and 15. The graph depicts that the temperature decreases when the values of Pr increase. This is due to the fact that a higher Pr fluid has relatively

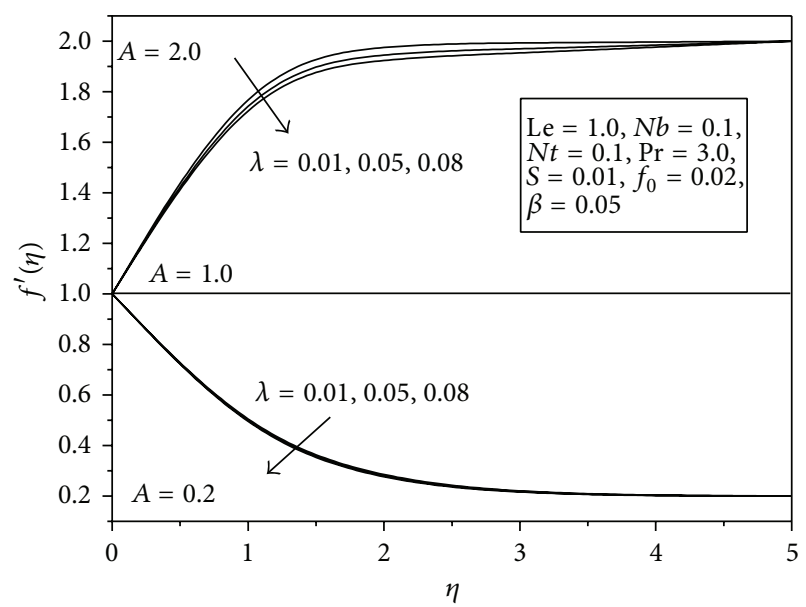

FIGURE 6: Velocity profile for different values of $\lambda$.

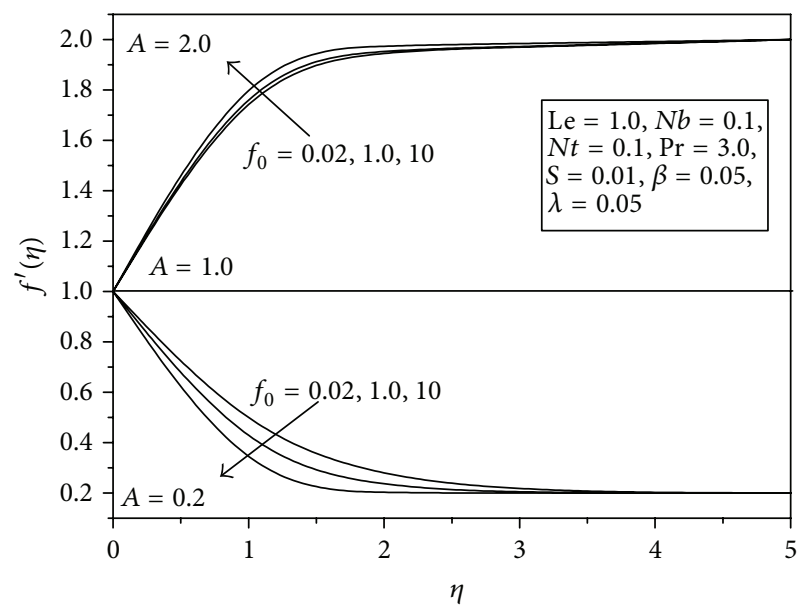

FIGURE 7: Velocity profile for different values of $f_{0}$. 
TABLE 3: Values of skin friction coefficient $-f^{\prime \prime}(0)$, Nusselt number $-\theta^{\prime}(0)$, and Sherwood number $-\phi^{\prime}(0)$ for different values of the physical parameters with $A=0.2$.

\begin{tabular}{|c|c|c|c|c|c|c|c|c|c|c|}
\hline$\beta$ & $\lambda$ & $f_{0}$ & Le & $\mathrm{Nb}$ & $N t$ & $\operatorname{Pr}$ & $S$ & $-f^{\prime \prime}(0)$ & $-\phi^{\prime}(0)$ & $-\theta^{\prime}(0)$ \\
\hline 0.05 & 0.05 & 0.02 & 1 & 0.1 & 0.1 & 3 & 0.1 & 0.9299 & 0.8437 & 0.8342 \\
\hline 1 & & & & & & & & 0.6836 & 0.8918 & 0.8785 \\
\hline 2 & & & & & & & & 0.5639 & 0.9156 & 0.8993 \\
\hline \multirow[t]{3}{*}{0.05} & 0.05 & 0.02 & 1 & 0.1 & 0.1 & 3 & 0.1 & 0.9299 & 0.8437 & 0.8342 \\
\hline & 1 & & & & & & & 1.3202 & 0.7776 & 0.7640 \\
\hline & 2 & & & & & & & 1.6397 & 0.7348 & 0.7077 \\
\hline \multirow[t]{3}{*}{0.05} & 0.05 & 0 & 1 & 0.1 & 0.1 & 3 & 0.1 & 0.9219 & 0.8354 & 0.8019 \\
\hline & & 1 & & & & & & 1.3479 & 1.1947 & 2.7517 \\
\hline & & 2 & & & & & & 1.7526 & 1.6380 & 4.9918 \\
\hline \multirow[t]{3}{*}{0.05} & 0.05 & 0.02 & 1 & 0.1 & 0.1 & 3 & 0.1 & 0.9299 & 0.8437 & 0.8342 \\
\hline & & & 2 & & & & & 0.9299 & 1.5778 & 0.7897 \\
\hline & & & 3 & & & & & 0.9299 & 2.1199 & 0.7695 \\
\hline \multirow[t]{3}{*}{0.05} & 0.05 & 0.02 & 1 & 0.1 & 0.1 & 3 & 0.1 & 0.9299 & 0.8437 & 0.8342 \\
\hline & & & & 1 & & & & 0.9299 & 1.2511 & 0.0874 \\
\hline & & & & 2 & & & & 0.9299 & 1.2436 & 0.0298 \\
\hline \multirow[t]{3}{*}{0.05} & 0.05 & 0.02 & 1 & 0.1 & 0.1 & 3 & 0.1 & 0.9299 & 0.8437 & 0.8342 \\
\hline & & & & & 1 & & & 0.9299 & 1.1108 & 0.3028 \\
\hline & & & & & 2 & & & 0.9299 & 3.1595 & 0.0844 \\
\hline \multirow[t]{3}{*}{0.05} & 0.05 & 0.02 & 1 & 0.1 & 0.1 & 1 & 0.1 & 0.9299 & 0.3671 & 0.5026 \\
\hline & & & & & & 2 & & 0.9299 & 0.6057 & 0.7138 \\
\hline & & & & & & 3 & & 0.9299 & 0.8437 & 0.8342 \\
\hline \multirow[t]{3}{*}{0.05} & 0.05 & 0.02 & 1 & 0.1 & 0.1 & 3 & -0.1 & 0.9299 & 0.6449 & 1.0726 \\
\hline & & & & & & & 0 & 0.9299 & 0.7404 & 0.9586 \\
\hline & & & & & & & 0.1 & 0.9299 & 0.8437 & 0.8342 \\
\hline
\end{tabular}

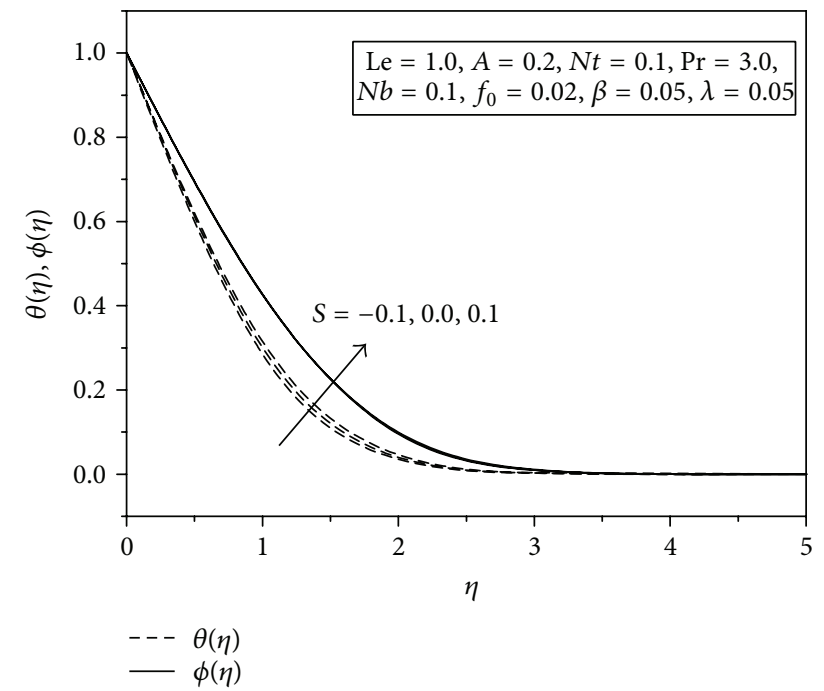

FiguRE 8: Temperature profile for different values of $S$.

low thermal conductivity, which reduces conduction and thereby the thermal boundary layer thickness, and as a result temperature decreases. We note that $N b, N t, \mathrm{Pr}$, and $S$ have no influence on the flow field, which is clear from (7). From

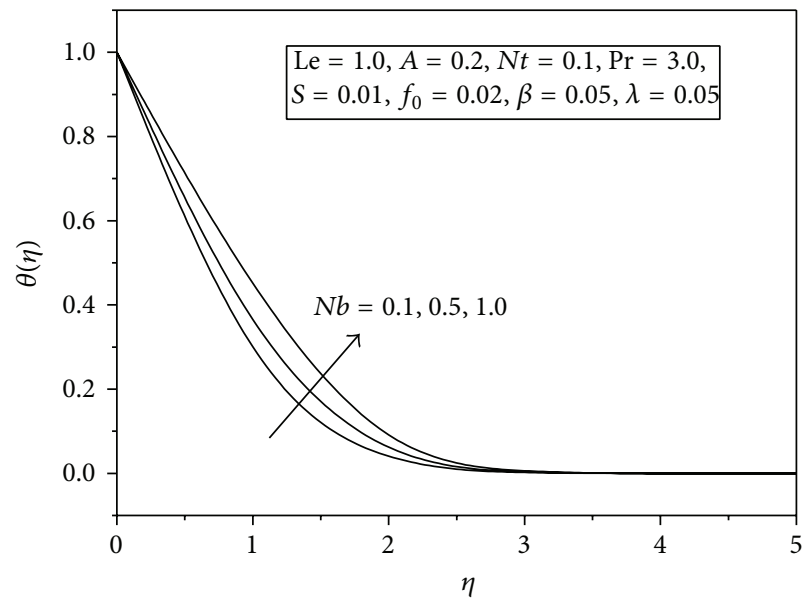

FIGURE 9: Temperature profile for different values of $\mathrm{Nb}$.

Table 3 one can see that the skin friction coefficient $f^{\prime \prime}(0)$ is negative at $A<1$. Physically, negative value of $f^{\prime \prime}(0)$ means the surface exerts a drag force on the fluid, and positive value means the opposite. This is not surprising since, in the present problem, we consider the case of a stretching sheet, which induces the flow. Negative value of $\theta^{\prime}(0)$ means the heat flows 
TABLE 4: Values of skin friction coefficient $f^{\prime \prime}(0)$, Nusselt number $-\theta^{\prime}(0)$, and Sherwood number $-\phi^{\prime}(0)$ for different values of the physical parameters with $A=2.0$.

\begin{tabular}{|c|c|c|c|c|c|c|c|c|c|c|}
\hline$\beta$ & $\lambda$ & $f_{0}$ & Le & $\mathrm{Nb}$ & $N t$ & $\operatorname{Pr}$ & $S$ & $f^{\prime \prime}(0)$ & $-\phi^{\prime}(0)$ & $-\theta^{\prime}(0)$ \\
\hline 0.05 & 0.05 & 0.02 & 1 & 0.1 & 0.1 & 3 & 0.1 & 1.9625 & 1.2545 & 1.1880 \\
\hline 1 & & & & & & & & 1.7009 & 1.2204 & 1.1565 \\
\hline 2 & & & & & & & & 1.4597 & 1.2022 & 1.1385 \\
\hline \multirow[t]{3}{*}{0.05} & 0.05 & 0.02 & 1 & 0.1 & 0.1 & 3 & 0.1 & 1.9625 & 1.2545 & 1.1880 \\
\hline & 1 & & & & & & & 1.0659 & 1.1603 & 1.1056 \\
\hline & 2 & & & & & & & 0.4741 & 1.0993 & 1.0491 \\
\hline \multirow[t]{3}{*}{0.05} & 0.05 & 0 & 1 & 0.1 & 0.1 & 3 & 0.1 & 1.9530 & 1.2453 & 1.1579 \\
\hline & & 1 & & & & & & 2.3962 & 1.5971 & 2.9677 \\
\hline & & 2 & & & & & & 2.7461 & 1.9483 & 5.1165 \\
\hline \multirow[t]{3}{*}{0.05} & 0.05 & 0.02 & 1 & 0.1 & 0.1 & 3 & 0.1 & 1.9625 & 1.2545 & 1.1880 \\
\hline & & & 2 & & & & & 1.9625 & 2.0475 & 1.1350 \\
\hline & & & 3 & & & & & 1.9625 & 2.6191 & 1.1091 \\
\hline \multirow[t]{3}{*}{0.05} & 0.05 & 0.02 & 1 & 0.1 & 0.1 & 3 & 0.1 & 1.9625 & 1.2545 & 1.1880 \\
\hline & & & & 1 & & & & 1.9625 & 1.6833 & 0.1720 \\
\hline & & & & 2 & & & & 1.9625 & 1.6664 & 0.0108 \\
\hline \multirow[t]{3}{*}{0.05} & 0.05 & 0.02 & 1 & 0.1 & 0.1 & 3 & 0.1 & 1.9625 & 1.2545 & 1.1880 \\
\hline & & & & & 1 & & & 1.9625 & 3.0176 & 0.4412 \\
\hline & & & & & 2 & & & 1.9625 & 6.5682 & 0.1676 \\
\hline \multirow[t]{3}{*}{0.05} & 0.05 & 0.02 & 1 & 0.1 & 0.1 & 1 & 0.1 & 1.9625 & 0.6307 & 0.8591 \\
\hline & & & & & & 2 & & 1.9625 & 0.9589 & 1.0804 \\
\hline & & & & & & 3 & & 1.9625 & 1.2545 & 1.1880 \\
\hline \multirow[t]{3}{*}{0.05} & 0.05 & 0.02 & 1 & 0.1 & 0.1 & 3 & -0.1 & 1.9625 & 1.1225 & 1.3437 \\
\hline & & & & & & & 0 & 1.9625 & 1.1876 & 1.2671 \\
\hline & & & & & & & 0.1 & 1.9625 & 1.2545 & 1.1880 \\
\hline
\end{tabular}

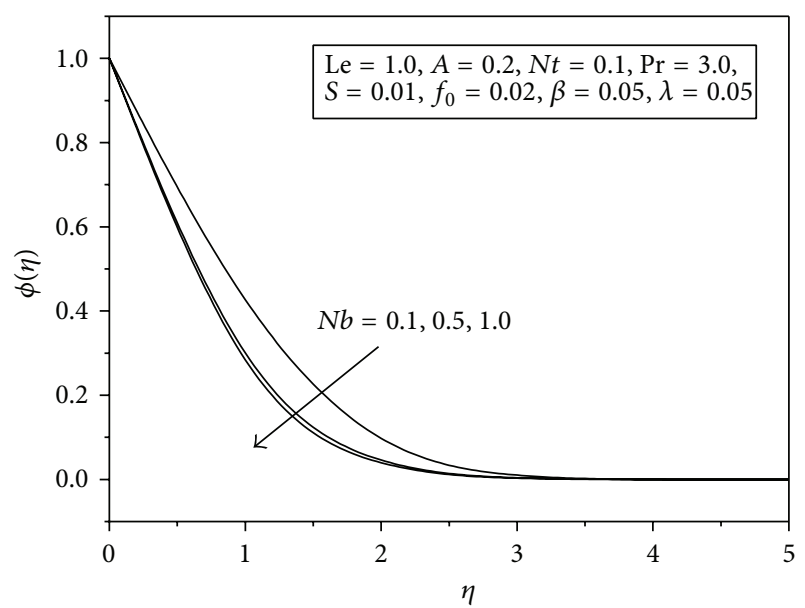

FIGURE 10: Concentration profile for different values of $\mathrm{Nb}$.

from the fluid to the solid surface. This is not surprising since the fluid is hotter than the solid surface.

\section{Conclusions}

In the present investigation, the influence of the different parameters on the velocity, temperature, and concentration profiles is illustrated and discussed. The numerical results

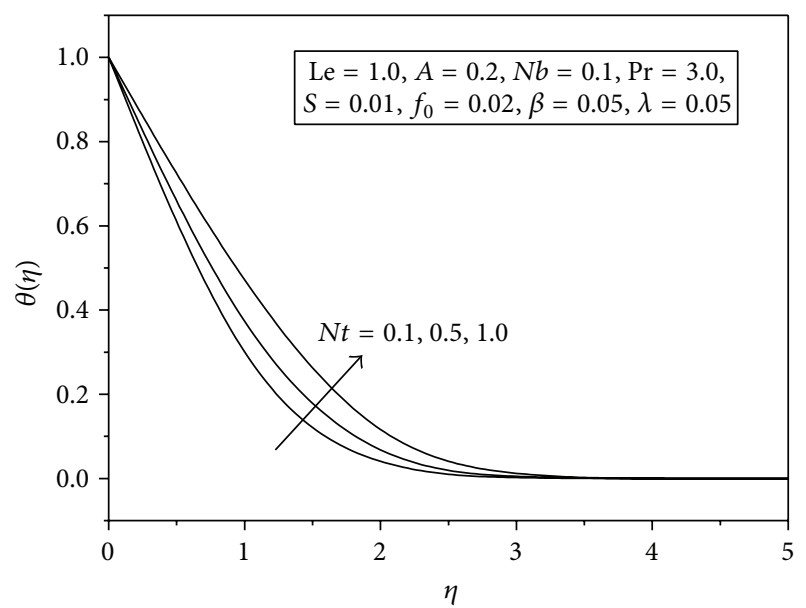

FIGURE 11: Temperature profile for different values of $N t$.

give a view towards understanding the response characteristics of the Jeffrey fluid in the presence of nanoparticles and heat generation/absorption. It is found that boundary layer is formed when $A>1$; on the other hand inverted boundary layer is formed when $A<1$. Some results of thermal characteristics at the wall are usually analyzed from the numerical results and the same are documented in Tables 3 and 4. Analyzing this table, it reveals that the effect 


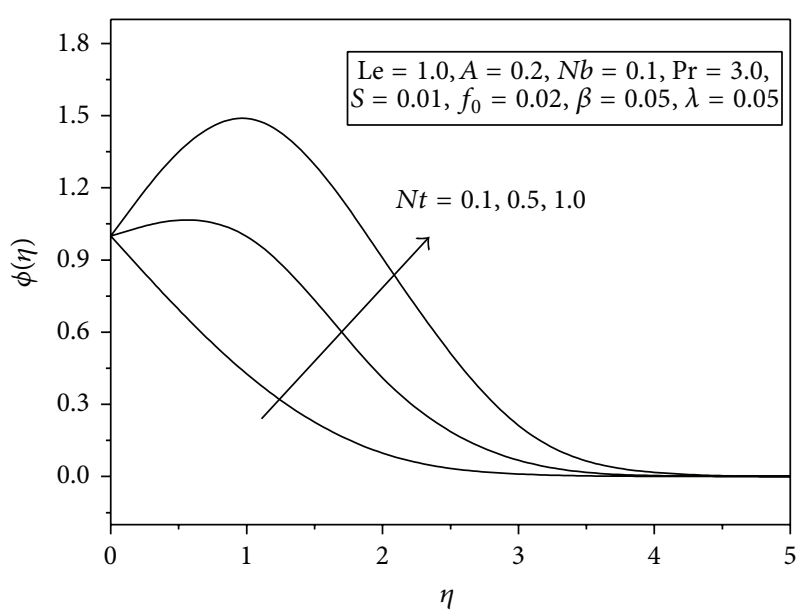

FIGURE 12: Concentration profile for different values of $\mathrm{Nt}$.

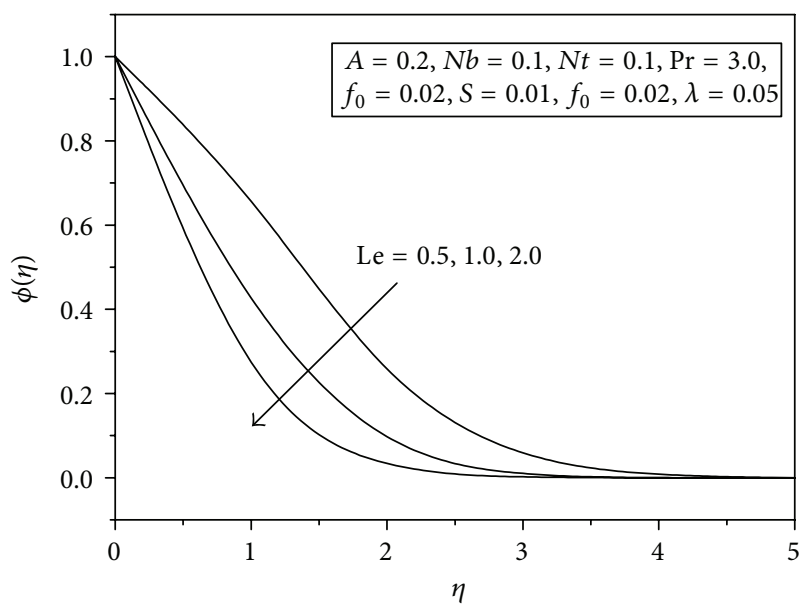

Figure 13: Concentration profile for different values of Le.

of increasing the values of $f_{0}$ and $\operatorname{Pr}$ is to decrease the $-\theta^{\prime}(0)$ and $-\phi^{\prime}(0)$ and the effect of increasing the values of $\lambda$, Le, $N b$, $N t$, and $S$ is to increase the $-\theta^{\prime}(0)$ and $-\phi^{\prime}(0)$. Also one can observe that there is no change in $-f^{\prime \prime}(0)$, when Le, $N b, N t$, $\operatorname{Pr}$, and $S$ vary.

\section{Nomenclature}
A: $\quad$ Velocity ratio
c: $\quad$ Stretching rate
$C_{f}:$ Skin friction
$c_{p}:$ Specific heat
$C$ : $\quad$ Nanoparticle volume fraction
$D_{B}$ : Brownian diffusion coefficient
$D_{T}$ : Thermophoresis diffusion coefficient
$N b$ : Brownian motion parameter
$N t$ : Thermophoresis parameter
$N u_{x}$ : Local Nusselt number
Le: Lewis number
Pr: Prandtl number
$Q_{0}$ : Dimensional heat generation/absorption coefficient

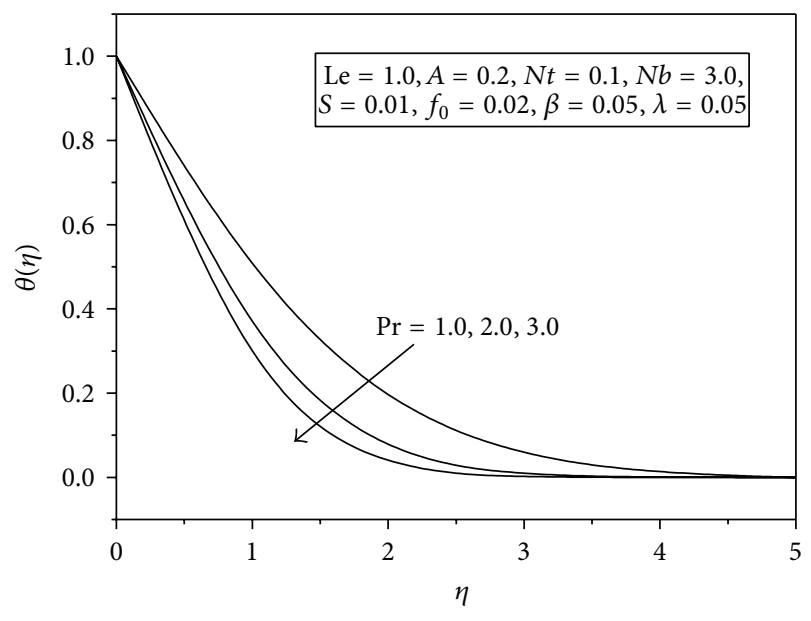

FIGURE 14: Temperature profile for different values of Pr.

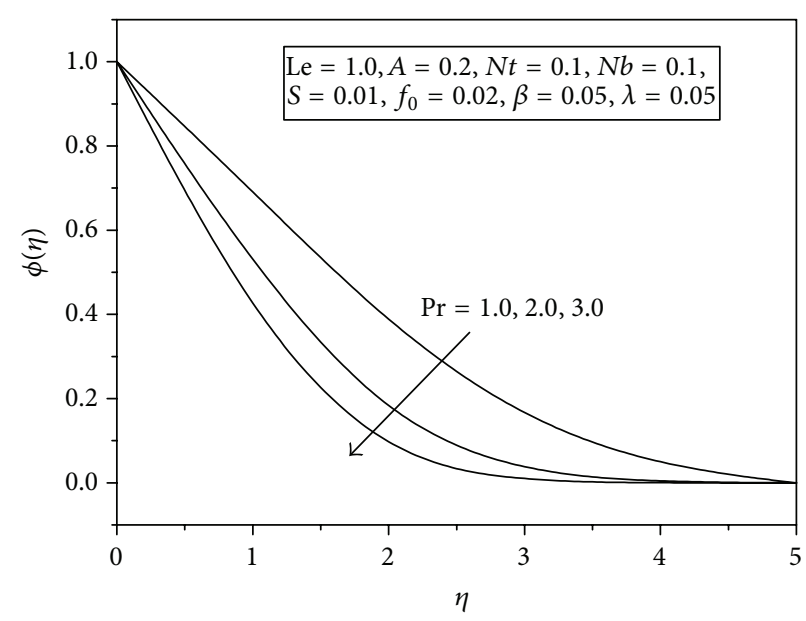

FIGURE 15: Concentration profile for different values of Pr.

S: Heat generation/absorption

$S h_{x}$ : Local Sherwood number

$T: \quad$ Temperature of the fluid

$T_{w}$ : Temperature at the wall

$T_{\infty}$ : Ambient fluid temperature

$u, v$ : Velocity components along $x$ and $y$ directions

$U_{w}$ : Stretching sheet velocity

$U_{\infty}$ : Free stream velocity

$x$ : Coordinate along the stretching sheet

$y$ : Distance normal to the stretching sheet.

\section{Greek Symbols}

$\nu$ : Kinematic viscosity

$\varphi$ : Rescaled nanoparticle volume fraction

$\rho_{f}$ : Density of the base fluid

$\rho_{p}$ : Density of the particles

$\lambda$ : Relaxation to retardation times

$\lambda_{1}$ : Relaxation time 


\author{
$\beta$ : Deborah number \\ $\theta$ : Dimensionless temperature \\ $\eta$ : Similarity variable \\ $\alpha$ : Thermal diffusivity \\ $\tau_{w}:$ Wall shearing stress.
}

\section{Conflict of Interests}

The author declares that there is no conflict of interests regarding the publication of this paper.

\section{Acknowledgments}

The author would like to express his appreciation to the referees for their valuable comments. The implementation of their suggestions has significantly improved the technical content of the paper.

\section{References}

[1] T. Hayat, N. Ahmad, and N. Ali, "Effects of an endoscope and magnetic field on the peristalsis involving Jeffrey fluid," Communications in Nonlinear Science and Numerical Simulation, vol. 13, no. 8, pp. 1581-1591, 2008.

[2] M. Kothandapani and S. Srinivas, "Peristaltic transport of a Jeffrey fluid under the effect of magnetic field in an asymmetric channel," International Journal of Non-Linear Mechanics, vol. 43, no. 9, pp. 915-924, 2008.

[3] B. C. Sakiadis, "Boundary-layer behavior on continuous solid surfaces: I. Boundary-layer equations for two-dimensional and axisymmetric flow," AIChE Journal, vol. 7, pp. 26-28, 1961.

[4] L. J. Crane, "Flow past a stretching plate," Zeitschrift für Angewandte Mathematik und Physik ZAMP, vol. 21, no. 4, pp. 645-647, 1970.

[5] K. Hiemenz, "Die grenzschicht an einem in dengleich formigen flussigkeitsstrom eingetauchten geraden kreiszlinder," Dingler's Polytech Journal, vol. 326, pp. 321-328, 1911.

[6] T. R. Mahapatra and A. S. Gupta, "Magnetohydrodynamic stagnation-point flow towards a stretching sheet," Acta Mechanica, vol. 152, no. 1-4, pp. 191-196, 2001.

[7] T. R. Mahapatra and A. S. Gupta, "Heat transfer in stagnationpoint flow towards a stretching sheet," Heat and Mass Transfer, vol. 38, no. 6, pp. 517-521, 2002.

[8] T. Ray Mahapatra, S. K. Nandy, and A. S. Gupta, "Magnetohydrodynamic stagnation-point flow of a power-law fluid towards a stretching surface," International Journal of NonLinear Mechanics, vol. 44, no. 2, pp. 123-128, 2009.

[9] A. Postelnicu, T. Groşan, and I. Pop, “The effect of variable viscosity on forced convection flow past a horizontal flat plate in a porous medium with internal heat generation," Mechanics Research Communications, vol. 28, no. 3, pp. 331-337, 2001.

[10] M. S. Abel, P. G. Siddheshwar, and M. M. Nandeppanavar, "Heat transfer in a viscoelastic boundary layer flow over a stretching sheet with viscous dissipation and non-uniform heat source," International Journal of Heat and Mass Transfer, vol. 50, no. 5-6, pp. 960-966, 2007.

[11] S. U. S. Choi, "Enhancing thermal conductivity of fluids with nanoparticles," in Proceedings of the ASME International Mechanical Engineering Congress \& Exhibition, vol. 66 of FED 231/MD, pp. 99-105, San Francisco, Calif, USA, November 1995.
[12] A. V. Kuznetsov and D. A. Nield, "Natural convective boundarylayer flow of a nanofluid past a vertical plate," International Journal of Thermal Sciences, vol. 49, no. 2, pp. 243-247, 2010.

[13] W. A. Khan and I. Pop, "Boundary-layer flow of a nanofluid past a stretching sheet," International Journal of Heat and Mass Transfer, vol. 53, no. 11-12, pp. 2477-2483, 2010.

[14] M. Mustafa, T. Hayat, I. Pop, S. Asghar, and S. Obaidat, "Stagnation-point flow of a nanofluid towards a stretching sheet," International Journal of Heat and Mass Transfer, vol. 54, no. 25-26, pp. 5588-5594, 2011.

[15] A. Alsaedi, M. Awais, and T. Hayat, "Effects of heat generation/absorption on stagnation point flow of nanofluid over a surface with convective boundary conditions," Communications in Nonlinear Science and Numerical Simulation, vol. 17, no. 11, pp. 4210-4223, 2012.

[16] M. M. Rahman, M. A. Al-Lawatia, I. A. Eltayeb, and N. Al-Salti, "Hydromagnetic slip flow of water based nanofluids past a wedge with convective surface in the presence of heat generation (or) absorption," International Journal of Thermal Sciences, vol. 57, pp. 172-182, 2012.

[17] S. K. Nandy and T. R. Mahapatra, "Effects of slip and heat generation/absorption on MHD stagnation flow of nanofluid past a stretching/shrinking surface with convective boundary conditions," International Journal of Heat and Mass Transfer, vol. 64, pp. 1091-1100, 2013.

[18] O. D. Makinde, W. A. Khan, and Z. H. Khan, "Buoyancy effects on MHD stagnation point flow and heat transfer of a nanofluid past a convectively heated stretching/shrinking sheet," International Journal of Heat and Mass Transfer, vol. 62, no. 1, pp. 526-533, 2013.

[19] W. Ibrahim, B. Shankar, and M. M. Nandeppanavar, "MHD stagnation point flow and heat transfer due to nanofluid towards a stretching sheet," International Journal of Heat and Mass Transfer, vol. 56, no. 1-2, pp. 1-9, 2013.

[20] G. K. Ramesh and B. J. Gireesha, "Influence of heat source/sink on a Maxwell fluid over a stretching surface with convective boundary condition in the presence of nanoparticles," Ain Shams Engineering Journal, vol. 5, pp. 991-998, 2014.

[21] S. Nadeem, R. Ul Haq, and Z. H. Khan, "Numerical solution of non-Newtonian nanofluid flow over a stretching sheet," Applied Nanoscience, vol. 4, no. 5, pp. 625-631, 2014.

[22] S. Nadeem, R. Mehmood, and N. S. Akbar, "Non-orthogonal stagnation point flow of a nano non-Newtonian fluid towards a stretching surface with heat transfer," International Journal of Heat and Mass Transfer, vol. 57, no. 2, pp. 679-689, 2013.

[23] P. Loganathan and C. Vimala, "MHD boundary layer flow of a nanofluid over an exponentially stretching sheet in the presence of radiation," Heat Transfer-Asian Research, vol. 43, no. 4, pp. 321-331, 2014.

[24] I. Anwar, S. Shafie, and M. Z. Salleh, "Radiation effect on MHD stagnation-point flow of a nanofluid over an exponentially stretching sheet," Walailak Journal of Science and Technology, vol. 11, no. 7, pp. 569-591, 2014.

[25] G. K. Ramesh, A. J. Chamkha, and B. J. Gireesha, "Magnetohydrodynamic flow of a non-Newtonian nanofluid over an impermeable surface with heat generation/absorption," Journal of Nanofluids, vol. 3, no. 1, pp. 78-84, 2014.

[26] T. Hayat, T. Javed, and Z. Abbas, "MHD flow of a micropolar fluid near a stagnation-point towards a non-linear stretching surface," Nonlinear Analysis: Real World Applications, vol. 10, no. 3, pp. 1514-1526, 2009. 
[27] T. Hayat, Z. Iqbal, M. Mustafa, and A. Alsaedi, "Stagnationpoint flow of Jeffrey fluid with melting heat transfer and Soret and Dufour effects," International Journal of Numerical Methods for Heat and Fluid Flow, vol. 24, no. 2, Article ID 17105513, pp. 402-418, 2014.

[28] F. M. Hady, F. S. Ibrahim, S. M. Abdel-Gaied, and M. R. Eid, "Effect of heat generation/absorption on natural convective boundary-layer flow from a vertical cone embedded in a porous medium filled with a non-Newtonian nanofluid," International Communications in Heat and Mass Transfer, vol. 38, no. 10, pp. 1414-1420, 2011. 

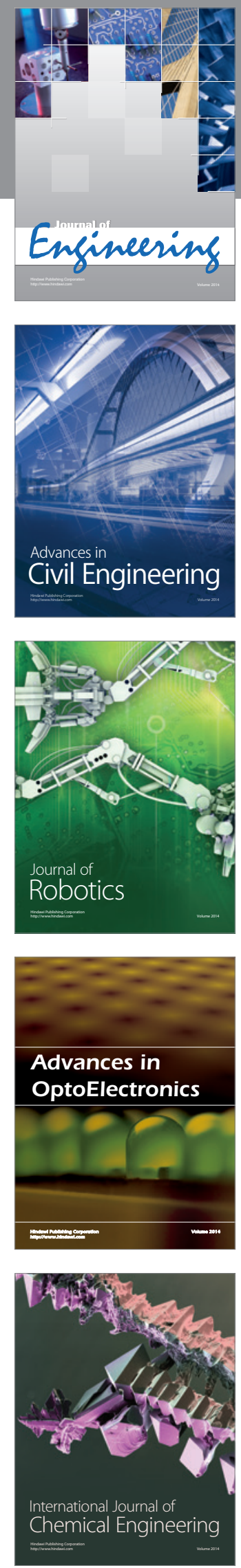

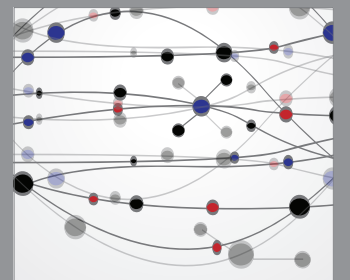

The Scientific World Journal
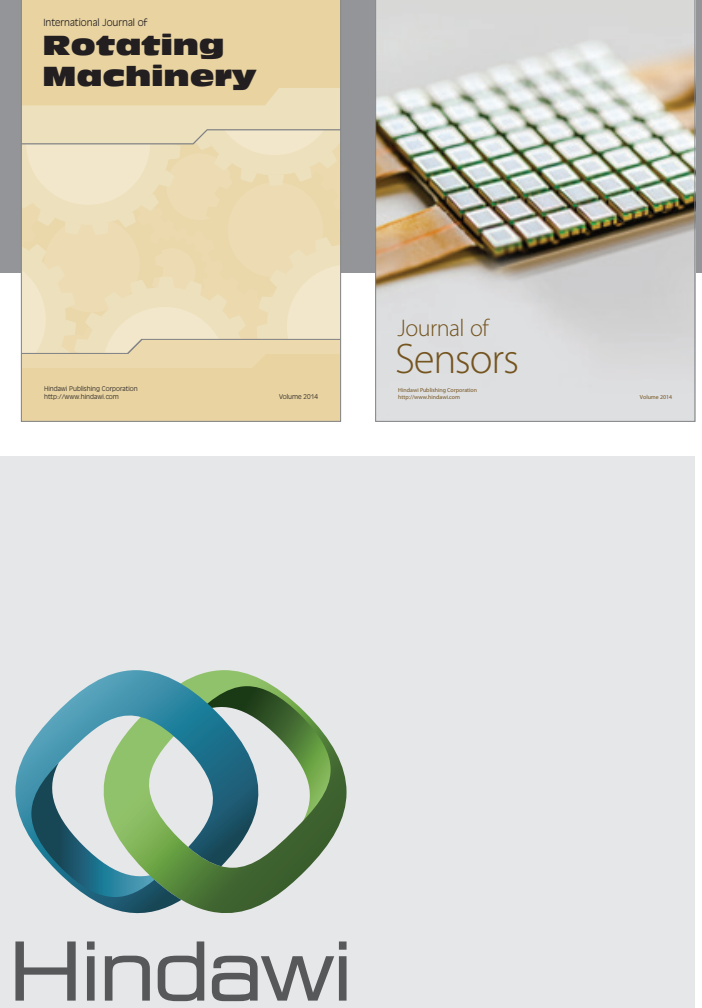

Submit your manuscripts at http://www.hindawi.com
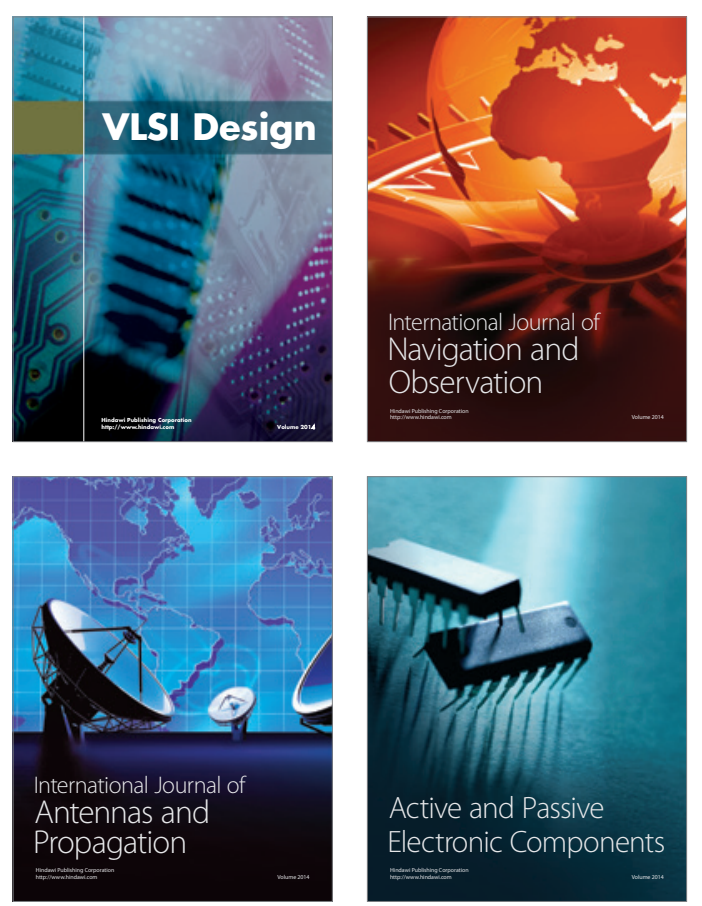
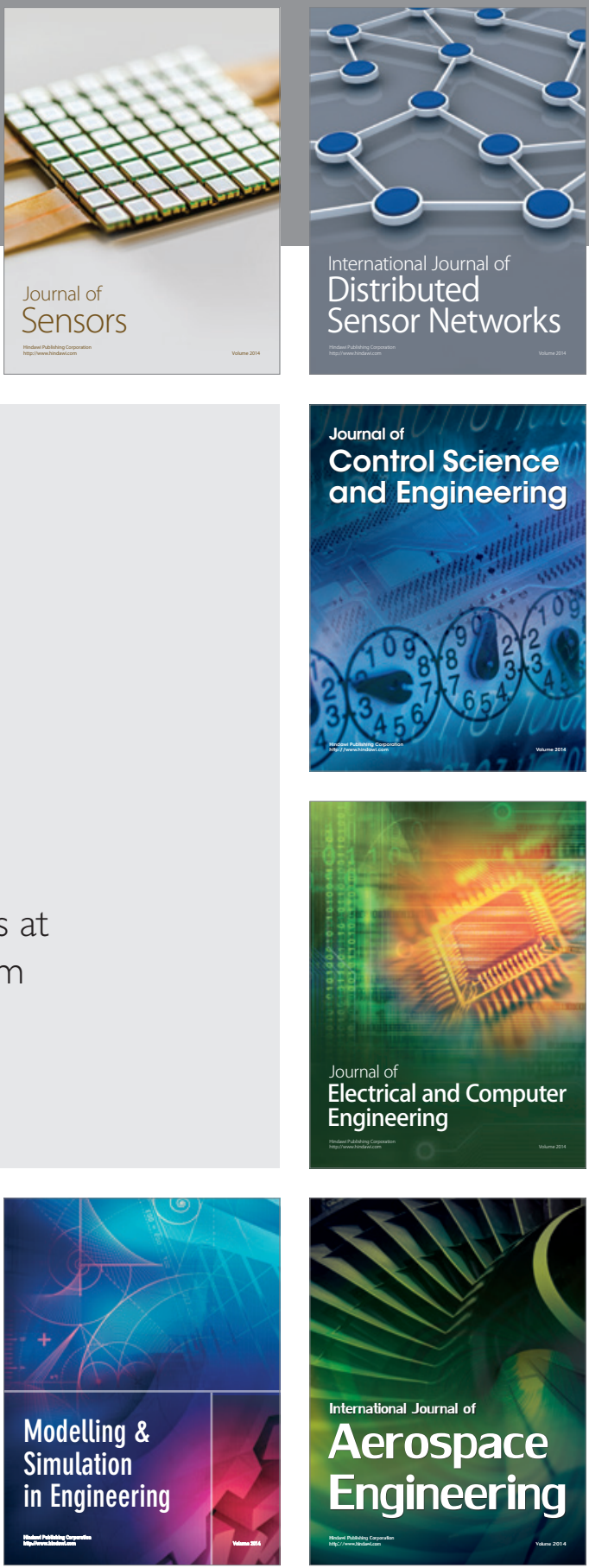

Journal of

Control Science

and Engineering
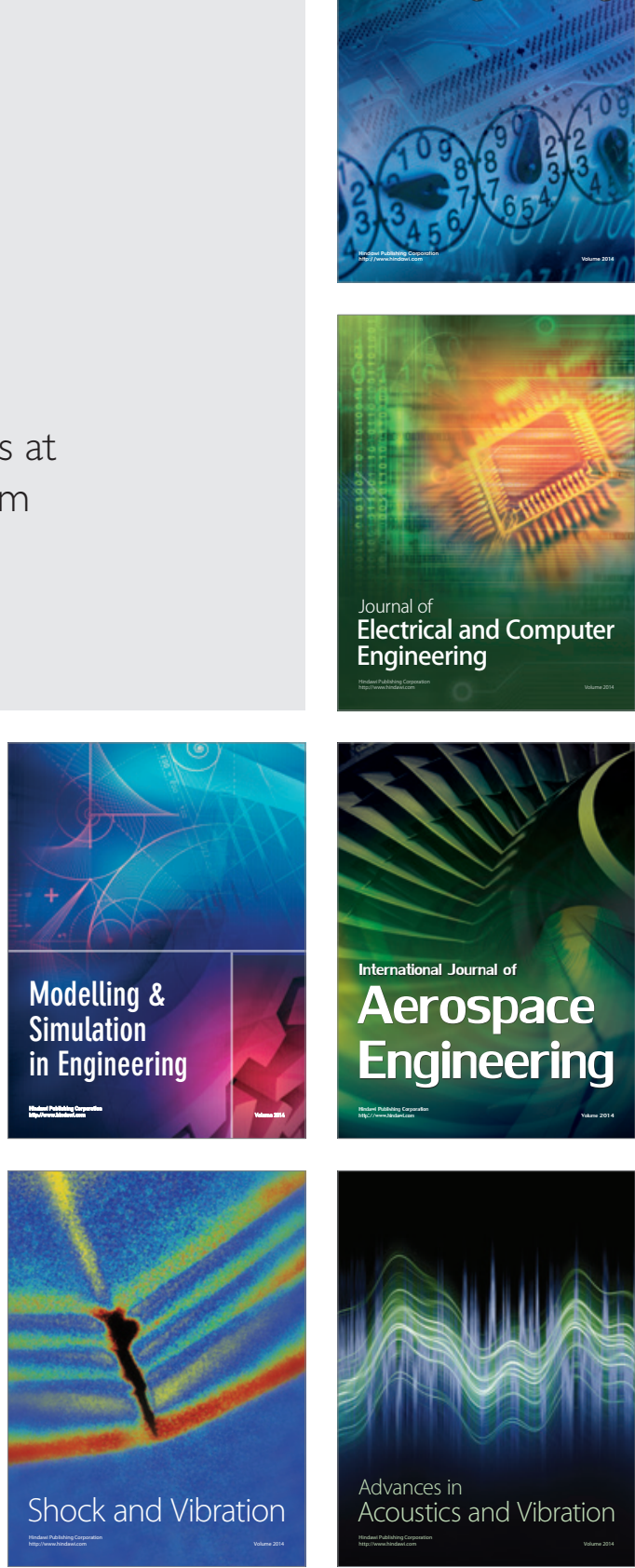